\title{
On unlikely intersections of complex varieties with tori
}

\author{
by \\ E. Bombieri (Princeton, NJ), D. Masser (Basel) \\ and U. ZANnier (Pisa)
}

To Wolfgang Schmidt with the very best of birthday wishes

1. Introduction. In the last several years there has been much investigation of what happens when an algebraic variety in an algebraic group is intersected with the union of all algebraic subgroups of a given dimension. We confine ourselves here to the multiplicative case; that is, the algebraic group is $\mathbb{G}_{m}^{n}$. There are several different forms of the main conjecture in the subject, and they are most naturally formulated over the complex numbers $\mathbb{C}$. However, the proofs up to now have always started with an algebraic variety defined over the algebraic numbers $\overline{\mathbb{Q}}$. In this note we present a general principle that enables the results over $\mathbb{C}$ to be deduced from the results over $\overline{\mathbb{Q}}$. This yields new results for curves and varieties of dimension $n-2$ (see Corollaries 1 and 2 below).

To classify the progress so far achieved over $\overline{\mathbb{Q}}$, we introduce integers $r \geq 0, s \geq 1$ and we consider varieties $\mathcal{X}$ of dimension $r$ in $\mathbb{G}_{m}^{r+s}$; thus $s$ is the codimension. We intersect with the union $\mathcal{H}_{s-1}$ of all algebraic subgroups of dimension $s-1$. For greater generality we shall henceforth work over an arbitrary field $K$ of zero characteristic with algebraic closure $\bar{K}$.

Regarding the exact form of the results, there are now several versions in the literature. For the moment we restrict ourselves to the following, which involves no preliminary definitions.

Conjecture. For every variety $\mathcal{X}$ of dimension $r$ in $\mathbb{G}_{m}^{r+s}$ defined over $K$ and irreducible over $\bar{K}$, there is a finite union $\mathcal{U}=\mathcal{U}(\mathcal{X})$ of proper algebraic subgroups of $\mathbb{G}_{m}^{r+s}$ such that $\mathcal{X} \cap \mathcal{H}_{s-1}$ is contained in $\mathcal{U}$.

2000 Mathematics Subject Classification: 11G35, 11G50, 14G25, 14J20.

Key words and phrases: intersections of algebraic varieties with algebraic subgroups, specialization. 
When $s=2$, this corresponds to Theorem 1.6 of [BMZ3, p. 6]. Its form is modelled on results and conjectures first due to Schinzel, and it indicates how these sorts of considerations can be extended to arbitrary dimension and codimension.

We postpone to an Appendix a detailed discussion of the other versions in the literature. The first to appear is due to Zilber [Z], also in the context of semiabelian varieties. Then Pink $[\mathrm{P}]$ suggested a version which could be interpreted also in the context of Shimura varieties. And the present authors in [BMZ3] gave versions in the context of $\mathbb{G}_{m}^{n}$.

To state our main result in a simple way, we may think of a picture with horizontal $r$-axis and vertical $s$-axis. Then the horizontal axis $s=0$ is meaningless for our problem, and the vertical axis $r=0$ represents only trivial results about single points. The horizontal line $s=1$ is the case of hypersurfaces, and the vertical line $r=1$ represents the case of curves. Similarly $s=2$ gives the case of codimension 2, and $r=2$ the case of surfaces. And so on. Now for integers $R \geq 0, S \geq 1$ we say that the Conjecture for $K$ holds on the $(R, S)$-rectangle if, for all integers $r, s$ with $0 \leq r \leq R, 1 \leq s \leq S$, it holds for every variety $\mathcal{X}$ of dimension $r$ in $\mathbb{G}_{m}^{r+s}$ defined over $K$ and irreducible over $\bar{K}$.

ThEOREM. Suppose that for some $R, S$ the Conjecture holds for $\overline{\mathbb{Q}}$ on the $(R, S)$-rectangle. Then the Conjecture holds for $\mathbb{C}$ on the $(R, S)$-rectangle.

Analogous results are true for the versions due to Zilber and to the present authors. In fact, we will show in the Appendix that these versions and the Conjecture, when restricted to any fixed $K$ and any fixed $(R, S)$ rectangle, are all equivalent.

For Zilber's version, Theorem 1 of [Z, p. 29] is similar to our Theorem but with unrestricted dimension and codimension; this can be regarded as the limiting case of our result with $R=S=\infty$, so that in the picture the whole quadrant is involved.

Here are some consequences of our result.

First, the horizontal line $s=1$ is the case of torsion points in hypersurfaces. This is known classically over $\overline{\mathbb{Q}}$. Taking $S=1$ yields the extension to $\mathbb{C}$; also probably known, and anyway easy to deduce just by ordinary specialization arguments.

Next, the vertical line $r=1$ is the case of curves. This was recently proved over $\overline{\mathbb{Q}}$ by Maurin $[\mathrm{M}]$. Taking $R=1$ yields the extension to $\mathbb{C}$, which is new. We state this explicitly as follows.

Corollary 1. For $n \geq 2$ let $\mathcal{C}$ be a complex curve in $\mathbb{G}_{m}^{n}$. Then the points of $\mathcal{C} \cap \mathcal{H}_{n-2}$ lie in a finite union of proper algebraic subgroups of $\mathbb{G}_{m}^{n}$. 
An example is the following, which can already be deduced from our Theorem for $S=4$ and the Corollary of [BMZ2, p. 2248]: there are only finitely many complex numbers $x \neq 0,1, \pi$ such that there are two independent multiplicative relations between

$$
2, \pi, x, x-1, x-\pi
$$

( $x=\pi / 2$ is one of them).

Note that for curves $\mathcal{X}=\mathcal{C}$ not lying in arbitrary translates of proper algebraic subgroups we already proved the Conjecture for $\mathbb{C}$ in our paper [BMZ1] using specialization arguments of a more elaborate nature; and the methods of the present article can be used to provide an alternative more natural proof.

Then the line $s=2$ is the case of varieties of codimension 2. Over $\overline{\mathbb{Q}}$ this was done recently in [BMZ3]; so taking $S=2$ gives once more the extension to $\mathbb{C}$, also new.

Corollary 2. For $n \geq 2$ let $\mathcal{D}$ be a complex variety of dimension $n-2$ in $\mathbb{G}_{m}^{n}$. Then the points of $\mathcal{D} \cap \mathcal{H}_{1}$ lie in a finite union of proper algebraic subgroups of $\mathbb{G}_{m}^{n}$.

Here is an example for threefolds $R=3$. Consider the set $X$ in $\mathbb{C}^{5}$ of all $\left(x_{1}, x_{2}, x_{3}, x_{4}, x_{5}\right)$ satisfying

$$
x_{1}+x_{2}+x_{3}+x_{4}+x_{5}=5 \pi, \quad x_{1} x_{2} x_{3} x_{4} x_{5}=\pi^{5}
$$

such that there are four independent multiplicative relations between $x_{1}, x_{2}, x_{3}, x_{4}, x_{5}$. Then $X$ lies in a finite union of proper algebraic subgroups of $\mathbb{G}_{m}^{5}$. In fact, these can be analyzed further to prove that $X$ is finite (it contains $(\pi, \pi, \pi, \pi, \pi)$ of course).

The line $r=2$ is the case of surfaces, about which not so much is known. In our paper [BMZ4] we proved some things about planes over $\overline{\mathbb{Q}}$. With the help of Maurin's result, these can be sharpened to fill in the line $r=2$ for non-degenerate planes over $\overline{\mathbb{Q}}$. So it seems likely that the arguments of the present note will lead to the extension to non-degenerate planes over $\mathbb{C}$.

Some words about the proof of our Theorem. A variety $\mathcal{X}$ defined over $\mathbb{C}$ is of course defined over some field finitely generated over $\mathbb{Q}$, say $\mathbb{Q}\left(t_{1}, \ldots, t_{e}\right)$. By thinking of the complex numbers $t_{1}, \ldots, t_{e}$ as extra variables, we can regard the defining equations as corresponding to a variety $\widetilde{\mathcal{X}}$ defined over $\mathbb{Q}$. This sort of thing is also done by Zilber [Z]. A point $P$ in $\mathcal{X} \cap \mathcal{H}_{s-1}$ corresponds to a subvariety $\mathcal{Y}_{P}$ of $\widetilde{\mathcal{X}}$ which is anomalous in the sense of [BMZ3], at least if $\mathcal{X}$ is not defined over $\overline{\mathbb{Q}}$. In fact, it may well be "over-anomalous" in a more general sense. The main geometric result of [BMZ3] gives a structure theorem for anomalous subvarieties which can easily be generalized, and applying this to $\mathcal{Y}_{P}$ yields after a few calculations the desired conclusions for $P$. 
2. Preliminaries. The following technical observation prevents the points of $\mathcal{X}$ acquiring extra multiplicative relations when regarded as points of $\widetilde{\mathcal{X}}$.

LEMMA 1. Given a positive integer $n$ and a finitely generated field extension $K / k$ in zero characteristic of transcendence degree $d \geq 1$, there is a finite collection $\mathcal{T}$ of sets of generators for $K / k$ with the following property. Let $x_{1}, \ldots, x_{n}$ be non-zero elements of any extension of $\bar{K}$. Then there is a set $\left\{t_{1}, \ldots, t_{e}\right\}$ from $\mathcal{T}$ whose elements are non-zero and multiplicatively independent of $x_{1}, \ldots, x_{n}$ modulo $\bar{k}^{*}$.

Proof. We start by noting that if $u_{1}, \ldots, u_{N}$ are any non-zero elements of $\bar{K}$ multiplicatively independent modulo $\bar{k}^{*}$, and $v$ in $\bar{K}$ is transcendental over $k$, then we can find an integer $j$ with $0 \leq j \leq N$ such that $u_{1}, \ldots, u_{N}, v+j$ are multiplicatively independent modulo $\bar{k}^{*}$. This is an easy argument based on the trivial fact that $v, v+1, \ldots, v+N$ are multiplicatively independent modulo $\bar{k}^{*}$.

Select a transcendence basis $\left(u_{1}, \ldots, u_{d}\right)$ of $K / k$. It is an equally trivial fact that the $D=(n+1) d$ elements

$$
u_{1}, \ldots, u_{d}, u_{1}+1, \ldots, u_{d}+1, \ldots, u_{1}+n, \ldots, u_{d}+n
$$

are multiplicatively independent modulo $\bar{k}^{*}$. Take any $v$ transcendental over $k$ with $K=k\left(u_{1}, \ldots, u_{d}, v\right)$. There is $j_{0}$ with $0 \leq j_{0} \leq D$ such that

$$
u_{1}, \ldots, u_{d}, u_{1}+1, \ldots, u_{d}+1, \ldots, u_{1}+n, \ldots, u_{d}+n, v+j_{0}
$$

are multiplicatively independent modulo $\bar{k}^{*}$. Then there is $j_{1}$ with $0 \leq j_{1} \leq$ $D+1$ such that

$$
u_{1}, \ldots, u_{d}, u_{1}+1, \ldots, u_{d}+1, \ldots, u_{1}+n, \ldots, u_{d}+n, v+j_{0}, v+j_{1}
$$

are multiplicatively independent modulo $\bar{k}^{*}$. And so on, until $j_{n}$ with $0 \leq$ $j_{n} \leq D+n$ such that

$$
u_{1}, \ldots, u_{d}, u_{1}+1, \ldots, u_{d}+1, \ldots, u_{1}+n, \ldots, u_{d}+n, v+j_{0}, \ldots, v+j_{n}
$$

are multiplicatively independent modulo $\bar{k}^{*}$. Take $\mathcal{T}$ as the set of $\left\{u_{1}+i\right.$, $\left.\ldots, u_{d}+i, v+j_{i}\right\}(i=0, \ldots, n)$ and correspondingly $\mathbf{t}_{i}=\left(u_{1}+i, \ldots\right.$, $\left.u_{d}+i, v+j_{i}\right)(i=0, \ldots, n)$ in $\mathbb{G}_{m}^{d+1}$. If the assertion of the lemma is false for some $\mathbf{x}=\left(x_{1}, \ldots, x_{n}\right)$ in $\mathbb{G}_{m}^{n}$, then for each $i$ there is $\mathbf{p}_{i}$ in $\mathbb{Z}^{n}$ and non-zero $\mathbf{q}_{i}$ in $\mathbb{Z}^{d+1}$ such that $\mathbf{x}^{\mathbf{p}_{i}} \mathbf{t}_{i}^{\mathbf{q}_{i}}$, in the usual vector exponential notation, lies in $\bar{k}$. Now the $\mathbf{x}^{\mathbf{p}_{i}}(i=0, \ldots, n)$ are certainly multiplicatively dependent, and it would follow that the quantities (1) are multiplicatively dependent modulo $\bar{k}^{*}$; a contradiction. Here we get the value $e=d+1$, clearly best possible.

In [BMZ3] we made repeated use of the Fibre Dimension Theorem. The following gives a slightly more precise conclusion. 
LEMMA 2. Let $\varphi$ be a morphism from the irreducible variety $\mathcal{V}$ to the irreducible variety $\mathcal{W}$. For an integer $m$, let $\mathcal{V}_{m}$ be the set of all $v$ in $\mathcal{V}$ such that $\varphi^{-1}(\varphi(v))$ has dimension at least $m$ at $v$. Then the Zariski closure of $\varphi\left(\mathcal{V}_{m}\right)$ has dimension at most $\operatorname{dim} \mathcal{V}-m$.

Proof. The idea is simply to consider the restriction of $\varphi$ from $\varphi^{-1}\left(\varphi\left(\mathcal{V}_{m}\right)\right)$ to $\varphi\left(\mathcal{V}_{m}\right)$, but with minor technical adjustments because these sets might not be irreducible or even closed.

From the standard Fibre Dimension Theorem (see for example FDT(c) of [BMZ3, p. 9], which does not require $\varphi$ to be dominant; or also the Semicontinuity Theorem of $\left[\mathrm{D}\right.$, p. 228]) we know that $\mathcal{V}_{m}$ is closed in $\mathcal{V}$. Accordingly, let $\mathcal{V}^{0}$ be any component of $\mathcal{V}_{m}$. It suffices to show that the Zariski closure $\widetilde{\mathcal{W}}$ of $\varphi\left(\mathcal{V}^{0}\right)$ has $\operatorname{dim} \widetilde{\mathcal{W}} \leq \operatorname{dim} \mathcal{V}-m$.

Now $\varphi\left(\mathcal{V}^{0}\right)$ contains a non-empty Zariski open set $\mathcal{U}^{0}$. For each $u$ in $\mathcal{U}^{0}$ we have $u=\varphi(v)$ for some $v$ in $\mathcal{V}^{0} \subseteq \mathcal{V}_{m}$ and so $\varphi^{-1}(u)=\varphi^{-1}(\varphi(v))$ has a component $\mathcal{V}_{v}$ through $v$ with $\operatorname{dim} \mathcal{V}_{v} \geq m$. This $\mathcal{V}_{v}$ lies in $\varphi^{-1}\left(\mathcal{U}^{0}\right)$ and therefore in one of the components of the Zariski closure of $\varphi^{-1}\left(\mathcal{U}^{0}\right)$ in $\mathcal{V}$. Thus we can find one of these components, say $\widetilde{\mathcal{V}}$, such that $\mathcal{V}_{v}$ lies in $\widetilde{\mathcal{V}}$ for a dense subset $S$ of $u=\varphi(v)$ in $\mathcal{U}^{0}$. Now consider the restriction $\widetilde{\varphi}$ of $\varphi$ from $\widetilde{\mathcal{V}}$ to $\widetilde{\mathcal{W}}$; both sets here are irreducible and the map is dominant because $\widetilde{\varphi}(\widetilde{\mathcal{V}})$ contains $S$. So by the standard Fibre Dimension Theorem (see for example FDT(b) of [BMZ3, p. 9]; or also part (b) of the first Theorem of $\left[\mathrm{D}\right.$, p. 228] ) there is a non-empty Zariski open subset $\mathcal{U}^{00}$ of $\mathcal{U}^{0}$ such that $\operatorname{dim} \widetilde{\varphi}^{-1}(u)=\operatorname{dim} \widetilde{\mathcal{V}}-\operatorname{dim} \widetilde{\mathcal{W}}$ for all $u$ in $\mathcal{U}^{00}$. Now $\mathcal{U}^{00}$ meets the dense set $S$ and so at a common point we have $\operatorname{dim} \widetilde{\varphi}^{-1}(u) \geq \operatorname{dim} \mathcal{V}_{v} \geq m$. It follows that

$$
\operatorname{dim} \widetilde{\mathcal{W}} \leq \operatorname{dim} \widetilde{\mathcal{V}}-m \leq \operatorname{dim} \mathcal{V}-m
$$

as required.

We next record a simple remark about fields of definition.

LEMma 3. Let $\mathcal{V}$ be an absolutely irreducible variety not defined over a field $k$. Then the points of $\mathcal{V}$ defined over $k$ are not Zariski dense in $\mathcal{V}$.

Proof. Consider the set $S$ of points of $\mathcal{V}$ defined over $k$. Then the Zariski closure $\mathcal{Z}$ of $S$ is also defined over $k$. So $\mathcal{Z}$ cannot be $\mathcal{V}$. Thus it is a proper subvariety of $\mathcal{V}$.

The following lemma gives a mild strengthening of another result in [BMZ3]. As there, the degree is taken with respect to the natural embedding of $\mathbb{G}_{m}^{n}$ in projective $\mathbb{P}_{n}$. And as usual we use the word coset to mean a translate of an algebraic group.

Lemma 4. There is a constant $C=C(n)$, depending only on $n$, with the following property. Suppose $1 \leq h \leq n$ and the absolutely irreducible 
variety $\mathcal{X}$ in $\mathbb{G}_{m}^{n}$ lies in a coset of dimension $n-h$. Then there are linearly independent $\mathbf{a}_{1}, \ldots, \mathbf{a}_{h}$ in $\mathbb{Z}^{n}$ with $\mathbf{x}^{\mathbf{a}_{1}}, \ldots, \mathbf{x}^{\mathbf{a}_{h}}$ constant on $\mathcal{X}$ and

$$
\max \left\{\left|\mathbf{a}_{1}\right|, \ldots,\left|\mathbf{a}_{h}\right|\right\} \leq C(\operatorname{deg} \mathcal{X})^{n-1} .
$$

Proof. We use induction on $n$. The case $n=1$ is trivial. So suppose $n \geq 2$. By Lemma 3.2 of [BMZ3, p. 14], there is a non-zero $\mathbf{a}_{h}$ in $\mathbb{Z}^{n}$ satisfying the required bound, with $\mathrm{x}^{\mathbf{a}_{h}}$ constant on $\mathcal{X}$. We can suppose that the last coordinate of $\mathbf{a}_{h}$ is non-zero. The projection $\pi(\mathcal{X})$ down to $\mathbb{G}_{m}^{n-1}$ lies in a coset of dimension $(n-1)-(h-1)$ in $\mathbb{G}_{m}^{n-1}$. So by induction there are linearly independent $\mathbf{a}_{1}, \ldots, \mathbf{a}_{h-1}$ in $\mathbb{Z}^{n-1}=\mathbb{Z}^{n-1} \times\{0\}$ inside $\mathbb{Z}^{n-1} \times \mathbb{Z}=\mathbb{Z}^{n}$, with $\mathbf{x}^{\mathbf{a}_{1}}, \ldots, \mathbf{x}^{\mathbf{a}_{h-1}}$ constant on $\pi(\mathcal{X})$ (or $\mathcal{X}$ ) and

$$
\max \left\{\left|\mathbf{a}_{1}\right|, \ldots,\left|\mathbf{a}_{h-1}\right|\right\} \leq C(n-1)(\operatorname{deg} \pi(\mathcal{X}))^{n-2} .
$$

Now $\operatorname{deg} \pi(\mathcal{X}) \leq \operatorname{deg} \mathcal{X}$; see for example the Proposition of [D, p. 254] for $\mathcal{X}$ not a cone over the centre $(0, \ldots, 0)$ of projection (in the case of a cone we have equality), and the result follows, because $\mathbf{a}_{h}$ not in $\mathbb{Z}^{n-1}$ must be independent of $\mathbf{a}_{1}, \ldots, \mathbf{a}_{h-1}$ in $\mathbb{Z}^{n-1}$.

Let $\mathcal{X}$ be an irreducible variety in $\mathbb{G}_{m}^{n}$, and let $b \geq 1$ be an integer. We say that an irreducible subvariety $\mathcal{Y}$ of $\mathcal{X}$ is $b$-anomalous if it has positive dimension and lies in some coset of dimension $n-(b+\operatorname{dim} \mathcal{X})+\operatorname{dim} \mathcal{Y}$. The case $b=1$ corresponds to Definition 1.1 of [BMZ3, p. 3] of anomalous, the case $b=2$ could be termed superanomalous, the case $b=3$ would be superduperanomalous, and so on. We also say that $\mathcal{Y}$ is maximal $b$ anomalous if it is not contained in another $b$-anomalous subvariety of $\mathcal{X}$ of strictly bigger dimension. The following is a mild strengthening of the main geometric result of [BMZ3].

Lemma 5. Given $\Delta \geq 1$ there is a constant $D=D(n, \Delta)$, depending only on $n$ and $\Delta$, with the following property. Suppose $\mathcal{X}$ is a variety in $\mathbb{G}_{m}^{n}$ of degree $\Delta$ defined over $K$ and irreducible over $\bar{K}$. Then any maximal b-anomalous subvariety $\mathcal{Y}$ of $\mathcal{X}$ lies in a coset $\mathbf{g} H^{(0)}$ satisfying

$$
\operatorname{dim} H^{(0)}=n-(b+\operatorname{dim} \mathcal{X})+\operatorname{dim} \mathcal{Y}, \quad \operatorname{deg} H^{(0)} \leq D
$$

with $\mathbf{g}$ in $\mathbb{G}_{m}^{n}(\bar{K})$.

Proof. We use double induction on $n$ and the difference

$$
c=\operatorname{dim} \mathcal{X}-\operatorname{dim} \mathcal{Y} \text {. }
$$

This notation means that on a $b$-anomalous subvariety $\mathcal{Y}$ of $\mathcal{X}$ there are $b+c$ independent relations $\mathbf{x}^{\mathbf{a}}=\alpha$.

If $c=0$ then $\mathcal{Y}=\mathcal{X}$ and so the assertion to be proved is an immediate consequence of Lemma 4 above, because the degree of $H^{(0)}$ defined by $\mathbf{x}^{\mathbf{a}_{1}}=$ $\cdots=\mathbf{x}^{\mathbf{a}_{h}}=1$ is at most $C_{0}\left|\mathbf{a}_{1}\right| \cdots\left|\mathbf{a}_{h}\right|$ for some $C_{0}$ depending only on $n$. 
If $c \geq 1$ then take $\mathcal{Y}$ in $\mathcal{X}$ as in the present lemma. It is certainly anomalous in $\mathcal{X}$, so by Proposition 3.1 of [BMZ3, p. 13] there is a relation $\mathrm{x}^{\mathbf{a}}=\alpha$ on $\mathcal{Y}$ with $\mathbf{a} \neq 0$ bounded only in terms of $n$ and $\Delta$. After adjusting with an automorphism if necessary, we can suppose that this relation is $x_{n}=\alpha$ on $\mathcal{Y}$.

Suppose first that $x_{n} \neq \alpha$ on $\mathcal{X}$. Then $\mathcal{Y}$ lies in some component $\mathcal{X}^{\prime}$ of the intersection of $\mathcal{X}$ with $x_{n}=\alpha$, and $\operatorname{dim} \mathcal{X}^{\prime}=\operatorname{dim} \mathcal{X}-1$. We may now consider $\mathcal{Y}$ as $(b+1)$-anomalous in $\mathcal{X}^{\prime}$. This is because $\mathcal{Y}$ lies in a coset of dimension

$$
n-(b+\operatorname{dim} \mathcal{X})+\operatorname{dim} \mathcal{Y}=n-\left(b^{\prime}+\operatorname{dim} \mathcal{X}^{\prime}\right)+\operatorname{dim} \mathcal{Y}
$$

if we define $b^{\prime}=b+1$.

We claim that $\mathcal{Y}$ is maximal $(b+1)$-anomalous in $\mathcal{X}^{\prime}$. For a strictly larger $b^{\prime}$-anomalous subvariety $\mathcal{Z}$ in $\mathcal{X}^{\prime}$ would lie in a coset of dimension

$$
n-\left(b^{\prime}+\operatorname{dim} \mathcal{X}^{\prime}\right)+\operatorname{dim} \mathcal{Z}=n-(b+\operatorname{dim} \mathcal{X})+\operatorname{dim} \mathcal{Z} .
$$

As $\mathcal{Z}$ lies in $\mathcal{X}$ this would contradict the maximality of $\mathcal{Y}$ as a $b$-anomalous subvariety of $\mathcal{X}$.

Thus indeed $\mathcal{Y}$ is maximal $(b+1)$-anomalous in $\mathcal{X}^{\prime}$. And the new value $c^{\prime}$ is

$$
c^{\prime}=\operatorname{dim} \mathcal{X}^{\prime}-\operatorname{dim} \mathcal{Y}=\operatorname{dim} \mathcal{X}-1-\operatorname{dim} \mathcal{Y}=c-1 .
$$

Thus by $c$-induction there are $b^{\prime}+c^{\prime}=b+c$ bounded independent relations on $\mathcal{Y}$, which is what we need. Here we have to note that the bound depends only on the degree of $\mathcal{X}^{\prime}$, which by Bézout is in turn bounded in terms of $n$ and $\Delta$.

What if $x_{n}=\alpha$ on $\mathcal{X}$ ? Then both $\mathcal{X}$ and $\mathcal{Y}$ are essentially in $\mathbb{G}_{m}^{n-1}$ because the last coordinates are constant. We note that $b \neq 1$, as otherwise $\mathcal{X}$ would itself be $b$-anomalous in $\mathcal{X}$, contradicting the maximality of $\mathcal{Y}$ in view of $c \geq 1$. We may now consider $\pi(\mathcal{Y})$ as $(b-1)$-anomalous in $\pi(\mathcal{X})$ in $\mathbb{G}_{m}^{n-1}$. To see this, note as above that $\mathcal{Y}$ lies in a coset $J$ of dimension $n-(b+\operatorname{dim} \mathcal{X})+\operatorname{dim} \mathcal{Y} \leq n-1$. After exchanging $x_{n}=\alpha$ for one of the equations of $J$, we can assume that $x_{n}=\alpha$ on $J$ without altering the dimension. This secures $\operatorname{dim} \pi(J)=\operatorname{dim} J$, and then $\pi(\mathcal{Y})$ lies in $\pi(J)$ of dimension

$$
n-(b+\operatorname{dim} \mathcal{X})+\operatorname{dim} \mathcal{Y}=(n-1)-\left(b^{\prime}+\operatorname{dim} \pi(\mathcal{X})\right)+\operatorname{dim} \pi(\mathcal{Y})
$$

if we define this time $b^{\prime}=b-1$.

We claim that $\pi(\mathcal{Y})$ is maximal $(b-1)$-anomalous in $\pi(\mathcal{X})$. For a strictly larger $(b-1)$-anomalous $\mathcal{Z}$ in $\pi(\mathcal{X})$ would lie in a coset $J^{\prime}$ in $\mathbb{G}_{m}^{n-1}$ of dimension

$$
(n-1)-\left(b^{\prime}+\operatorname{dim} \pi(\mathcal{X})\right)+\operatorname{dim} \mathcal{Z}=n-(b+\operatorname{dim} \mathcal{X})+\operatorname{dim}(\mathcal{Z} \times\{\alpha\}) .
$$


Then $\mathcal{Y}$ would lie strictly within $\mathcal{Z} \times\{\alpha\}$ and also within $J^{\prime} \times\{\alpha\}$ of the same dimension as the above, again contradicting the maximality of $\mathcal{Y}$ as a $b$-anomalous subvariety of $\mathcal{X}$.

Thus indeed $\pi(\mathcal{Y})$ is maximal $(b-1)$-anomalous in $\pi(\mathcal{X})$. Now the $c$ has not decreased, but the ambient dimension has, and so this time it is $n$-induction that provides $b^{\prime}+c=b+c-1$ bounded independent relations on $\pi(\mathcal{Y})$ and so on $\mathcal{Y}$. We get an extra one via $x_{n}=\alpha$, of course. This completes the proof.

3. Proof of Theorem. For fixed $r \geq 0, s \geq 1$ and $K$ we denote the Conjecture by $\operatorname{Conj}(r, s ; K)$. It suffices to deduce $\operatorname{Conj}(r, s ; \mathbb{C})$ from all the $\operatorname{Conj}\left(r^{\prime}, s^{\prime} ; \overline{\mathbb{Q}}\right)$ for $r^{\prime}, s^{\prime}$ satisfying

$$
0 \leq r^{\prime} \leq r, \quad 1 \leq s^{\prime} \leq s
$$

In fact, it suffices to deduce $\operatorname{Conj}(r, s ; K)$ for any fixed $K$ which is finitely generated over $\mathbb{Q}$. And then we may even assume the validity of all the $\operatorname{Conj}\left(r^{\prime}, s^{\prime} ; K\right)$ for $r^{\prime}, s^{\prime}$ satisfying

$$
0 \leq r^{\prime} \leq r, \quad 1 \leq s^{\prime} \leq s, \quad r^{\prime}+s^{\prime}<r+s .
$$

Let $\mathcal{T}=\mathcal{T}(K / k)$ be the collection in Lemma 1 with $n=r+s$ and $k=\mathbb{Q}$.

Take $\mathcal{X}$ in $\mathbb{G}_{m}^{n}$ defined over $K$ and irreducible over $\bar{K}$. Let $d$ be the transcendence degree of $K$ over $\mathbb{Q}$. In proving the Conjecture for $\mathcal{X}$ we can assume not only that $d \geq 1$ but also that $d$ is minimal; in particular, $\mathcal{X}$ is not defined over $\overline{\mathbb{Q}}$.

We have to show that there is a finite union of proper algebraic subgroups which contains $\mathcal{X} \cap \mathcal{H}_{s-1}$. So let $P$ be any point of this intersection. For clarity we shall sometimes indicate the ambient dimension by writing $\mathcal{H}_{s-1}=\mathcal{H}_{s-1}^{(n)}$.

By Lemma 1 applied to the coordinates of $P$, we can choose a set $\left\{t_{1}, \ldots, t_{e}\right\}$ from $\mathcal{T}$ whose elements are multiplicatively independent of these coordinates modulo $\overline{\mathbb{Q}}^{*}$. Write $\mathbf{t}=\left(t_{1}, \ldots, t_{e}\right)$.

To $\mathcal{X}$ with coordinates $\mathbf{x}=\left(x_{1}, \ldots, x_{n}\right)$ in $\mathbb{G}_{m}^{n}$ we can associate a variety $\widetilde{\mathcal{X}}$ with coordinates $\widetilde{\mathbf{x}}=(\mathbf{x}, \mathbf{t})=\left(x_{1}, \ldots, x_{n}, t_{1}, \ldots, t_{e}\right)$ in $\mathbb{G}_{m}^{\widetilde{n}}=\mathbb{G}_{m}^{n} \times \mathbb{G}_{m}^{e}$ with $\widetilde{n}=n+e$. This can be defined as a variety over $\mathbb{Q}$ by stipulating that if $\mathbf{x}$ is a generic point of $\mathcal{X}$ over $K=\mathbb{Q}\left(t_{1}, \ldots, t_{e}\right)$, then $\widetilde{\mathbf{x}}$ is a generic point of $\widetilde{\mathcal{X}}$ over $\mathbb{Q}$. Because $\mathcal{X}$ is irreducible over $\bar{K}$, it is easy to see that $\widetilde{\mathcal{X}}$ is irreducible over $\overline{\mathbb{Q}}$. Its dimension is $\widetilde{r}=r+d$; for example we can consider the tower of fields

$$
\mathbb{Q}, \mathbb{Q}\left(t_{1}, \ldots, t_{e}\right), \mathbb{Q}\left(x_{1}, \ldots, x_{n}, t_{1}, \ldots, t_{e}\right)
$$

and note that the successive relative transcendence degrees are $d$ and $r$. 
Our point $P$ in $\mathcal{X} \cap \mathcal{H}_{s-1}$ lies in some translate $\mathbf{z} H_{s-1}$ by a torsion point $\mathbf{z}$ of a connected algebraic subgroup $H_{s-1}$ in $\mathbb{G}_{m}^{n}$ of dimension $s-1$. It gives rise to a variety $\mathcal{Y}_{P}$ in $\widetilde{\mathcal{X}}$ the coordinates of whose generic point consist of the coordinates of $P$ together with $t_{1}, \ldots, t_{e}$. This $\mathcal{Y}_{P}$ lies in $\widetilde{\mathbf{z}} H_{s-1+e}$, where $\widetilde{\mathbf{z}}=\left(\mathbf{z},\{1\}^{e}\right)$ and $H_{s-1+e}=H_{s-1} \times \mathbb{G}_{m}^{e}$. The independent relations defining $\widetilde{\mathbf{z}} H_{s-1+e}$ can be taken as

$$
\mathbf{x}^{\mathbf{a}_{1}}=\zeta_{1}, \ldots, \mathbf{x}^{\mathbf{a}_{r+1}}=\zeta_{r+1}
$$

for roots of unity $\zeta_{1}, \ldots, \zeta_{r+1}$. Let $d^{*} \geq d$ be the dimension of $\mathcal{Y}_{P}$.

There is a maximal $h$ such that $\mathcal{Y}_{P}$ is contained in some translate by an element of $\mathbb{G}_{m}^{\widetilde{n}}(\overline{\mathbb{Q}})$ of some connected algebraic subgroup in $\mathbb{G}_{m}^{\widetilde{n}}$ of dimension $\widetilde{n}-h$; and clearly $\widetilde{n}-h \leq s-1+e$ so $h \geq n-s+1=r+1$. On the other hand, $h \leq n$ because the relations defining the subgroup cannot involve the generators, and so there are at most $n$ of them.

Now a simple calculation shows that $\mathcal{Y}_{P}$ is $b$-anomalous in $\tilde{\mathcal{X}}$ with

$$
b=h-r+d^{*}-d \geq 1 .
$$

Assume for the moment that $\mathcal{Y}_{P}$ is maximal $b$-anomalous. Since by Lemma 1 there are only finitely many possibilities for $\widetilde{\mathcal{X}}$, we deduce from Lemma 5 that $\mathcal{Y}_{P}$ lies in some translate $\mathbf{g} H_{\widetilde{n}-h}^{(0)}$ of some bounded connected algebraic subgroup $H_{\widetilde{n}-h}^{(0)}$ in $\mathbb{G}_{m}^{\widetilde{n}}$ of dimension $\widetilde{n}-h$; here $\mathbf{g}$ lies in $\mathbb{G}_{m}^{\widetilde{n}}(\overline{\mathbb{Q}})$. Intersecting further with $\widetilde{\mathbf{z}} H_{s-1+e}$ and using the maximality of $h$ we deduce that

$$
\mathbf{g} H_{\widetilde{n}-h}^{(0)} \subseteq \widetilde{\mathbf{z}} H_{s-1+e} .
$$

From the choice of transcendence basis the relations defining $\mathbf{g} H_{\widetilde{n}-h}^{(0)}$ do not involve $\mathbf{t}$.

Let us first dispose of the possibility $h=n$. Then $H_{\widetilde{n}-h}^{(0)}=\{1\}^{n} \times \mathbb{G}_{m}^{e}$. But this would imply that all the coordinates of our point $P$ are algebraic. And now Lemma 3 (with $k=\overline{\mathbb{Q}}$ ) implies that $P$ lies in some fixed proper subvariety $\mathcal{Z}$ of $\mathcal{X}$, because we assumed that $d \geq 1$ was minimal and so $\mathcal{X}$ is certainly not defined over $\overline{\mathbb{Q}}$. Therefore the projection $\mathcal{Z}^{\prime}$ of $\mathcal{Z}$ down to any $\mathbb{G}_{m}^{n-1}$ has dimension at most $r-1$. In case the actual dimension is less than $r-1$, we can embed $\mathcal{Z}^{\prime}$ in a fixed variety $\mathcal{X}^{\prime}$, still in $\mathbb{G}_{m}^{n-1}$, of dimension exactly $r^{\prime}=r-1$. The projection of $P$ lies in $\mathcal{H}_{s-1}=\mathcal{H}_{s-1}^{(n-1)}$. So by $\operatorname{Conj}\left(r^{\prime}, s^{\prime} ; K\right)$ with $s^{\prime}=s$ it must lie in a finite union of fixed proper algebraic subgroups of $\mathbb{G}_{m}^{n-1}$, and thus so does $P$ itself.

Thus we can assume $h<n$. Now after applying an automorphism of $\mathbb{G}_{m}^{n}$ we can take $H_{\widetilde{n}-h}^{(0)}=\{1\}^{h} \times \mathbb{G}_{m}^{n-h} \times \mathbb{G}_{m}^{e}$. From (3) we see that the relations (2) cannot involve $x_{h+1}, \ldots, x_{n}$. Thus the projection of $P$ to $\mathbb{G}_{m}^{h}$ lies in $\mathcal{H}_{h-r-1}=\mathcal{H}_{h-r-1}^{(h)}$. The projection of $\mathcal{X}$ to $\mathbb{G}_{m}^{h}$ has dimension at 
most $r$, and as before we can embed it in a fixed variety $\mathcal{X}^{\prime}$, still in $\mathbb{G}_{m}^{h}$, of dimension exactly $r^{\prime}=r$ and so $\operatorname{Conj}\left(r^{\prime}, s^{\prime} ; K\right)$ with $s^{\prime}=h-r<n-r=s$ gives again what we want.

But what if $\mathcal{Y}_{P}$ was not maximal $b$-anomalous? Then it is contained in a maximal $b$-anomalous subvariety $\mathcal{Z}_{P}$ in $\widetilde{\mathcal{X}}$, and the dimension of $\mathcal{Z}_{P}$ can be written as $\widetilde{n}-l$ for some $l$ satisfying $n-r+e-d \leq l \leq n-1+e-d^{*}$ (the possibility $l=n+e-d^{*}$ has in effect just been treated above). Write

$$
p=h-n+l+d^{*}-e \geq h-n+(n-r)=h-r \geq 1,
$$

so that also $p \leq h-1<n$. Then Lemma 5 shows that $\mathcal{Z}_{P}$ lies in some translate $\mathbf{g} H_{\widetilde{n}-p}^{(0)}$ of some bounded connected algebraic subgroup $H_{\widetilde{n}-p}^{(0)}$ in $\mathbb{G}_{m}^{\widetilde{n}}$ of dimension $\widetilde{n}-p$. As above, using the property in Lemma 1 we can suppose after applying an automorphism of $\mathbb{G}_{m}^{\widetilde{n}}$ that $\mathbf{g} H_{\widetilde{n}-p}^{(0)}$ is defined by

$$
x_{1}=\gamma_{1}, \ldots, x_{p}=\gamma_{p}
$$

with $\gamma_{1}, \ldots, \gamma_{p}$ algebraic.

These relations (4) then hold on $\mathcal{Y}_{P}$. And so do (2). Together they give $p+r+1 \geq h+1$ relations. But we know that there are no more than $h$ relations on $\mathcal{Y}_{P}$. Therefore there are at least

$$
q=p+r+1-h=l+r+1-n+d^{*}-e \geq 1
$$

relations

$$
b_{i 1} \mathbf{a}_{1}+\cdots+b_{i, r+1} \mathbf{a}_{r+1}=\left(a_{i 1}, \ldots, a_{i p}, 0, \ldots, 0\right) \quad(i=1, \ldots, q)
$$

in $\mathbb{Z}^{n}$, independent in the sense that the integer vectors $\left(b_{i 1}, \ldots, b_{i, r+1}\right.$, $\left.a_{i 1}, \ldots, a_{i p}\right) \quad(i=1, \ldots, q)$ are linearly independent, together with relations

$$
\gamma_{1}^{a_{i 1}} \ldots \gamma_{p}^{a_{i p}}=\eta_{i} \quad(i=1, \ldots, q)
$$

for roots of unity

$$
\eta_{i}=\zeta_{1}^{b_{i 1}} \cdots \zeta_{r+1}^{b_{i, r+1}} \quad(i=1, \ldots, q) .
$$

In fact, the vectors $\left(a_{i 1}, \ldots, a_{i p}\right)(i=1, \ldots, q)$ are linearly independent. For a non-trivial relation

$$
\sum_{i=1}^{q} g_{i}\left(a_{i 1}, \ldots, a_{i p}\right)=0
$$

would lead via (5) to a relation $\sum_{j=1}^{r+1} w_{j} \mathbf{a}_{j}=0$ with $w_{j}=\sum_{i=1}^{q} g_{i} b_{i j}(j=$ $1, \ldots, r+1)$. Then the independence of the $\mathbf{a}_{j}$ in (2) would imply the vanishing of all the $w_{j}$; that is, the equations $\sum_{i=1}^{q} g_{i}\left(b_{i 1}, \ldots, b_{i, r+1}\right)=0$. This together with (8) would then contradict the independence of the relations (5).

We are going to examine the point $Q=\left(\gamma_{1}, \ldots, \gamma_{p}\right)$ in $\mathbb{G}_{m}^{p}$. It actually lies in some small variety in $\mathbb{G}_{m}^{p}$ defined over $\overline{\mathbb{Q}}$. In fact, with $\mathcal{V}=\widetilde{\mathcal{X}}$ and $\mathcal{W}=\mathbb{G}_{m}^{p}$ consider the map $\varphi$ from $\mathcal{V}$ to $\mathcal{W}$ defined by $\varphi(\mathbf{x}, \mathbf{t})=\left(x_{1}, \ldots, x_{p}\right)$. 
Above $Q$ we get at least the variety $\mathcal{Z}_{P}$, by (4). Thus $Q$ lies in $\varphi\left(\mathcal{V}_{m}\right)$ with $m=\operatorname{dim} \mathcal{Z}_{P}$. So it lies in the Zariski closure $\mathcal{Z}$ of $\varphi\left(\mathcal{V}_{m}\right)$, and by Lemma 2 we have

$\operatorname{dim} \mathcal{Z} \leq \operatorname{dim} \mathcal{V}-m=\widetilde{r}-(\widetilde{n}-l)=r-n+l+d-e \leq r-n+l+d^{*}-e$.

As before it may turn out that this is too small; so we embed $\mathcal{Z}$ in a fixed variety $\mathcal{X}^{\prime}$, still in $\mathbb{G}_{m}^{p}$ and defined over $\overline{\mathbb{Q}}$, with dimension exactly $r^{\prime}=$ $r-n+l+d^{*}-e<r$.

What is more, the equations (6) are $q$ independent multiplicative relations for $Q$. This means that $Q$ lies in $\mathcal{H}_{p-q}=\mathcal{H}_{p-q}^{(p)}$. Write $s^{\prime}=1+p-q=$ $h-r \leq s$; then we are in $\mathbb{G}_{m}^{p}$ with $p=r^{\prime}+s^{\prime}$. Thus we can appeal to $\operatorname{Conj}\left(r^{\prime}, s^{\prime} ; \overline{\mathbb{Q}}\right)$ to conclude that $Q$ lies in a bounded proper subgroup of $\mathbb{G}_{m}^{p}$. And so we have an additional relation

$$
\gamma_{1}^{a_{1}} \cdots \gamma_{p}^{a_{p}}=1
$$

with bounded integer exponents not all zero.

Finally, (9) and (4) lead to $x_{1}^{a_{1}} \cdots x_{p}^{a_{p}}=1$ on $\mathcal{Z}_{P}$. And lo and behold, we have our bounded proper subgroup containing $P$ ! The Theorem is therefore proved.

Appendix. We rename the Conjecture above as Conjecture 1. We proceed to list some other Conjectures $2-5$ which can be found in the literature. These various conjectures may well be equivalent; and we even expect them all to be true. At least we shall establish their equivalence on rectangles. This then means that our Theorem holds for each of these conjectures.

We start the list. We define a torsion coset as a translate of a connected algebraic group by a torsion point.

Conjecture 2. For every variety $\mathcal{X}$ of dimension $r$ in $\mathbb{G}_{m}^{r+s}$ defined over $K$ and irreducible over $\bar{K}$, there is a finite union $\mathcal{U}_{F}=\mathcal{U}_{F}(\mathcal{X})$ of torsion cosets $T$ of $\mathbb{G}_{m}^{r+s}$ with $\operatorname{dim}(\mathcal{X} \cap T) \geq \operatorname{dim} T-s+1$ such that $\mathcal{X} \cap \mathcal{H}_{s-1}=$ $\mathcal{X} \cap \mathcal{U}_{F} \cap \mathcal{H}_{s-1}$.

This corresponds to Theorem 1.5 of [BMZ3, p. 6] when $s=2$.

As in [BMZ3], we say that an irreducible subvariety $\mathcal{Y}$ of $\mathcal{X}$ is torsionanomalous if it is contained in an algebraic subgroup $H$ satisfying

$$
\operatorname{dim} \mathcal{Y}>\max \{0, \operatorname{dim} \mathcal{X}+\operatorname{dim} H-n\}=\max \{0, \operatorname{dim} H-s\} .
$$

Conjecture 3. For every variety $\mathcal{X}$ of dimension $r$ in $\mathbb{G}_{m}^{r+s}$ defined over $K$ and irreducible over $\bar{K}$, there is a finite collection $\Omega=\Omega(\mathcal{X})$ of torsion-anomalous subvarieties $\mathcal{Y}$ of $\mathcal{X}$ such that the intersection of $\mathcal{H}_{s-1}$ with $\mathcal{X}$ deprived of the members of $\Omega$ is finite.

For $s=2$ this can be found at the beginning of the proof of Theorem 1.7 of [BMZ3, p. 26] (misprinted there as Theorem 5.1). 
As in [BMZ3], we denote by $\mathcal{X}^{\text {ta }}$ what remains in $\mathcal{X}$ after removing all torsion-anomalous subvarieties $\mathcal{Y}$ of $\mathcal{X}$.

Conjecture 4. For every variety $\mathcal{X}$ of dimension $r$ in $\mathbb{G}_{m}^{r+s}$ defined over $K$ and irreducible over $\bar{K}$, the set $\mathcal{X}^{\text {ta }}$ is open in $\mathcal{X}$ and $\mathcal{X}^{\text {ta }} \cap \mathcal{H}_{s-1}$ is finite.

This is the combination of the Torsion Openness Conjecture and the Torsion Finiteness Conjecture of [BMZ3, p. 25], corresponding to Theorem 1.7 there when $s=2$.

Finally, when $T$ is a torsion coset we say that a component $\mathcal{Y}$ of $\mathcal{X} \cap T$ is atypical if

$$
\operatorname{dim} \mathcal{Y}>\operatorname{dim} \mathcal{X}+\operatorname{dim} T-n=\operatorname{dim} T-s .
$$

Conjecture 5. For every variety $\mathcal{X}$ of dimension $r$ in $\mathbb{G}_{m}^{r+s}$ defined over $K$ and irreducible over $\bar{K}$, there is a finite union $\mathcal{U}_{Z}=\mathcal{U}_{Z}(\mathcal{X})$ of proper algebraic subgroups of $\mathbb{G}_{m}^{r+s}$ such that for every torsion coset $T$ each atypical component of $\mathcal{X} \cap T$ is contained in $\mathcal{U}_{Z}$.

This corresponds to Zilber's Conjecture [Z], the first of its kind to be published.

As mentioned, we shall shortly prove that these are all equivalent to the original Conjecture 1 provided we work on rectangles. But for completeness we state a last variation.

Conjecture 6. For every variety $\mathcal{X}$ of dimension $r$ in $\mathbb{G}_{m}^{r+s}$ defined over $K$ and irreducible over $\bar{K}$ not lying in any proper algebraic subgroup of $\mathbb{G}_{m}^{r+s}$, the set $\mathcal{X} \cap \mathcal{H}_{s-1}$ is not Zariski dense in $\mathcal{X}$.

This corresponds to Pink's Conjecture $[\mathrm{P}]$. We do not know if it is equivalent to the others on rectangles. However, Ullmo has pointed out that it is equivalent to Conjecture 1 in the limiting situation $R=S=\infty$ of the whole quadrant. Thus in this situation all six conjectures are equivalent.

To prove the equivalence of Conjectures $1-5$ on rectangles, we argue cyclically around a pentagon in the natural order. We abbreviate Conjecture $i$ for fixed $r, s, K$ simply to $C_{i}(r, s)(i=1,2,3,4,5)$; here the field $K$ plays no role. And $C_{i}$ denotes $C_{i}(r, s)(i=1,2,3,4,5)$ on a rectangle defined by $0 \leq r \leq R, 1 \leq s \leq S$. We shall be somewhat concise in our proofs, freely using automorphisms and references to [BMZ3].

$C_{1} \Rightarrow C_{2}$. (Essentially as in the deduction of Theorem 1.4 from Theorem 1.6 in [BMZ3, p. 23].) Let $\mathcal{X}$ of dimension $r$ be in $\mathbb{G}_{m}^{n}$ for $n=r+s$, and let $P$ be any point of $\mathcal{X} \cap \mathcal{H}_{s-1}$. By $C_{1}(r, s)$ there exist rational integers $a_{1}, \ldots, a_{n}$ such that $P$ lies in the essentially fixed proper algebraic subgroup defined by $x_{1}^{a_{1}} \cdots x_{n}^{a_{n}}=1$. So it lies in some component $T_{n-1}$, which after 
the usual automorphism we can assume to be defined by $x_{n}=\zeta_{n}$ for a fixed root of unity $\zeta_{n}$.

If $x_{n}=\zeta_{n}$ on $\mathcal{X}$ then

$$
\operatorname{dim}\left(\mathcal{X} \cap T_{n-1}\right)=r=(n-1)-(s-1)=\operatorname{dim} T_{n-1}-s+1
$$

and so for this $P$ we will throw this $T_{n-1}$ into the collection $\mathcal{U}_{F}$ below.

If $x_{n} \neq \zeta_{n}$ on $\mathcal{X}$ then any component $\widetilde{\mathcal{X}}$ of $\mathcal{X} \cap T_{n-1}$ has the form $\tilde{\mathcal{X}}=\mathcal{X}^{\prime} \times\left\{\zeta_{n}\right\}$ for $\mathcal{X}^{\prime}$ in $\mathbb{G}_{m}^{n-1}$ with $\operatorname{dim} \mathcal{X}^{\prime} \leq r-1$. But the projection $\pi$ of $\mathcal{X}$ to the last coordinate is dominant and so the Fibre Dimension Theorem shows that $\operatorname{dim} \widetilde{\mathcal{X}} \geq r-1$. Thus $\operatorname{dim} \widetilde{\mathcal{X}}=\operatorname{dim} \mathcal{X}^{\prime}=r-1$.

We take $\widetilde{\mathcal{X}}$ as the component of $\mathcal{X} \cap T_{n-1}$ through $P$, which has the form $P=P^{\prime} \times\left\{\zeta_{n}\right\}$ for $P^{\prime}$ in $\mathbb{G}_{m}^{n-1}$. As $P^{\prime}$ lies in $\mathcal{X}^{\prime} \cap \mathcal{H}_{s-1}$ we can inductively use $C_{1}(r-1, s)$ or $C_{2}(r-1, s)$ to see that $P^{\prime}$ lies in one of a finite number of fixed torsion cosets $T^{\prime}$ in $\mathbb{G}_{m}^{n-1}$ with $\operatorname{dim}\left(\mathcal{X}^{\prime} \cap T^{\prime}\right) \geq \operatorname{dim} T^{\prime}-s+1$. Thus $P$ lies in $T=T^{\prime} \times\left\{\zeta_{n}\right\}$ itself in $T_{n-1}$; and now $\mathcal{X} \cap T=\left(\mathcal{X} \cap T_{n-1}\right) \cap T$ contains $\widetilde{\mathcal{X}} \cap T=\left(\mathcal{X}^{\prime} \cap T^{\prime}\right) \times\left\{\zeta_{n}\right\}$. Thus

$$
\operatorname{dim}(\mathcal{X} \cap T) \geq \operatorname{dim}\left(\mathcal{X}^{\prime} \cap T^{\prime}\right) \geq \operatorname{dim} T^{\prime}-s+1=\operatorname{dim} T-s+1 .
$$

We now see our collection $\mathcal{U}_{F}$, and since $P$ was arbitrary we have shown that $\mathcal{X} \cap \mathcal{H}_{s-1}$ lies in the union over $\mathcal{U}_{F}$ of the $(\mathcal{X} \cap T) \cap \mathcal{H}_{s-1}$. So clearly $\mathcal{X} \cap \mathcal{H}_{s-1}$ is this union, and $C_{2}(r, s)$ is proved.

$C_{2} \Rightarrow C_{3}$. (Essentially as in the first part of the proof of Theorem 1.7 of [BMZ3, p. 26], misprinted there as Theorem 5.1.) Let $\mathcal{X}$ of dimension $r$ be in $\mathbb{G}_{m}^{n}$ for $n=r+s$. We note in $C_{2}(r, s)$ that

$$
\operatorname{dim} T \leq s-1+\operatorname{dim}(\mathcal{X} \cap T) \leq s-1+\operatorname{dim} \mathcal{X}=n-1
$$

for each $T$ in $\mathcal{U}_{F}$. Thus by enlarging $T$ if necessary we can assume that it has dimension $n-1$ and that $\mathcal{X} \cap \mathcal{H}_{s-1}$ is contained in the union of the $(\mathcal{X} \cap T) \cap \mathcal{H}_{s-1}$.

Next, we claim that we can also assume that every component of each $\mathcal{X} \cap T$ has dimension $r-1$. Indeed, after an automorphism we can suppose that $T$ is defined by $x_{n}=\zeta_{n}$ for a root of unity $\zeta_{n}$. If the projection $\pi$ of $\mathcal{X}$ to the last coordinate is not dominant, then $x_{n}$ would be constant on $\mathcal{X}$. But then $x_{n}=\zeta_{n}$ on $\mathcal{X}$. This would mean that $\mathcal{X}$ itself is torsion-anomalous, and in this case $C_{3}(r, s)$ is trivially true with a single $\mathcal{Y}=\mathcal{X}$. So we can suppose that $\pi$ is dominant. Then the Fibre Dimension Theorem as above shows indeed that every component of $\mathcal{X} \cap T$ has dimension $r-1$.

Now the projection $\mathcal{X}^{\prime}$ of a component of $\mathcal{X} \cap T$ to $\mathbb{G}_{m}^{n-1}$ also has dimension $r-1$, and so $C_{2}(r-1, s)$ or $C_{3}(r-1, s)$ gives a finite collection $\Omega^{\prime}$ of torsion-anomalous subvarieties $\mathcal{Y}^{\prime}$ of $\mathcal{X}^{\prime}$ such that the intersection of $\mathcal{H}_{s-1}$ with $\mathcal{X}^{\prime}$ deprived of the members of $\Omega^{\prime}$ is finite. It is not difficult to see that each $\mathcal{Y}^{\prime} \times\left\{\zeta_{n}\right\}$ here is also torsion-anomalous in $\mathcal{X}$ in $\mathbb{G}_{m}^{n}$. For $\operatorname{dim} \mathcal{Y}^{\prime} \geq 1$ 
and $\mathcal{Y}^{\prime}$ lies in an algebraic subgroup $H$ of $\mathbb{G}_{m}^{n-1}$ satisfying

$$
\operatorname{dim} \mathcal{Y}^{\prime} \geq 1+\operatorname{dim} \mathcal{X}^{\prime}+\operatorname{dim} H-(n-1)=r+\operatorname{dim} H-n+1 .
$$

This is

$$
\operatorname{dim}\left(\mathcal{Y}^{\prime} \times\left\{\zeta_{n}\right\}\right) \geq r+\operatorname{dim} H-n+1=1+\operatorname{dim} \mathcal{X}+\operatorname{dim}\left(H \times\left\{\zeta_{n}\right\}\right)-n .
$$

Thus indeed $\mathcal{Y}^{\prime} \times\left\{\zeta_{n}\right\}$ is torsion-anomalous in $\mathcal{X}$ in $\mathbb{G}_{m}^{n}$. And so to get $\Omega(\mathcal{X})$ above it suffices to take the union of these after adjusting each one by the corresponding automorphism. This establishes $C_{3}(r, s)$.

$C_{3} \Rightarrow C_{4}$. (Essentially as in the second part of the proof of Theorem 1.7 of [BMZ3, p. 27].) Let $\mathcal{X}$ of dimension $r$ be in $\mathbb{G}_{m}^{n}$ for $n=r+s$, and let $\mathcal{Y}_{0}$ be any torsion-anomalous subvariety of $\mathcal{X}$. We observed just after the statement of the Torsion Finiteness Conjecture of [BMZ3, p. 25] that $\mathcal{Y}_{0} \cap \mathcal{H}_{s-1}$ is dense in $\mathcal{Y}_{0}$ (this is the argument which shows that every $\mathcal{Y}_{0}$ obstructs the finiteness of $\left.\mathcal{X} \cap \mathcal{H}_{s-1}\right)$. It follows that $\mathcal{Y}_{0}$ lies in the union of the $\mathcal{Y}$ in $\Omega$. Thus if $\mathcal{Y}_{0}$ was maximal torsion-anomalous, then it must be one of these $\mathcal{Y}$. This shows that there are only finitely many maximal torsion-anomalous subvarieties of $\mathcal{X}$; and now the proof of $C_{4}(r, s)$ is easily completed.

$C_{4} \Rightarrow C_{5}$. This can be found literally in [BMZ3, p. 29] just after the statement of Zilber's Conjecture.

$C_{5} \Rightarrow C_{1}$. (Suggested by a personal communication of Zilber.) Let $\mathcal{X}$ of dimension $r$ be in $\mathbb{G}_{m}^{n}$ for $n=r+s$, and let $P$ be any point of $\mathcal{X} \cap \mathcal{H}_{s-1}$. Then $P$ lies in some component $\mathcal{Y}$ of some $\mathcal{X} \cap H_{s-1}$ for an algebraic subgroup $H_{s-1}$ of dimension $s-1$. By definition $\mathcal{Y}$ is atypical. So $C_{5}(r, s)$ shows that $P$ lies in $\mathcal{U}_{Z}$. Thus we may take this as $\mathcal{U}$ in $C_{1}(r, s)$.

\section{References}

[BMZ1] E. Bombieri, D. Masser and U. Zannier, Finiteness results for multiplicatively dependent points on complex curves, Michigan Math. J. 51 (2003), 451-466.

[BMZ2] -, -, 一, Intersecting curves and algebraic subgroups: conjectures and more results, Trans. Amer. Math. Soc. 358 (2006), 2247-2257.

[BMZ3] -, - - - Anomalous subvarieties - structure theorems and applications, Int. Math. Res. Notices 2007, Article ID rnm057 (33 pages), doi: 10.1093/imrn/ rnm057.

[BMZ4] - - - - , Intersecting a plane with algebraic subgroups of multiplicative groups, Ann. Scuola Norm. Sup. Pisa Cl. Sci. (5) 7 (2008), 51-80.

[D] V. I. Danilov, Algebraic varieties and schemes, in: Algebraic Geometry I, I. R. Shafarevich (ed.), Encyclopaedia Math. Sci. 23, Springer, 1994, 167-297.

[M] G. Maurin, Conjecture de Zilber-Pink pour les courbes tracées sur des tores, Prépublication de l'Institut Fourier 696, 2007, 29 pp.

[P] R. Pink, A common generalization of the conjectures of André-Oort, ManinMumford, and Mordell-Lang, manuscript, 2005, 13 pp. 
[Z] B. Zilber, Exponential sums equations and the Schanuel conjecture, J. London Math. Soc. 65 (2002), 27-44.

School of Mathematics

Institute for Advanced Study

Princeton, NJ 08540, U.S.A.

E-mail: eb@math.ias.edu

Scuola Normale Superiore

Piazza Cavalieri 7

56126 Pisa, Italy

E-mail: u.zannier@sns.it
Mathematisches Institut

Universität Basel Rheinsprung 21

4051 Basel, Switzerland E-mail: David.Masser@unibas.ch

Received on 13.1.2008

and in revised form on 7.8.2008 